\title{
protection contre les ébranlements liés aux travaux de déroctage
}

par

\author{
A. Rozière \\ S.N.C.F.-Ouvrages d'Art, Paris
}

RESUME - Depuis quelques années, l'utilisation d'explosifs et d'engins de déroctage puissants s'est considérablement développée sur les chantiers de travaux publics. Les risques qui en découlent, et, en particulier les risques d'ébranlement ont conduit la SNCF à adopter une politique de limitation des vibrations produites, tant sur ses propres chantiers que sur les chantiers de tiers à proximité de ses emprises. Une procédure d'essais et de contrôle a été définie. Des laboratoires spécialisés ont été agréés pour l'exécution des mesures. Un petit nombre d'entreprises spécialisées ont été agréées pour l'exécution des tirs. Dans le cas particulier des chantiers de mise au gabarit ou de réfection de tunnels anciens, les problèmes sont très spécifiques. La mesure de vibrations à très courte distance des zones de tir est rendue difficile par l'étalement de celles-ci sur une très large bande de fréquences.

\section{1 - IMPORTANCE DU PROBLEME}

\subsection{Introduction}

L'utilisation de l'explosif sur les chantiers de chemin de fer a débuté dès l'origine de ceux-ci. avec les premiers terrassements et le creusement des premiers tunnels et s'est poursuivie pour les travaux neufs jusqu'à ce jour (chantiers du TGV). Certains chantiers d'abaissement de plateforme pour mise au gabarit électrification ont été également effectués à l'explosif après la dernière guerre. De nombreux chantiers de tiers (carrières, barrages, rectifications de routes) se sont également effectués depuis l'origine à proximité d'ouvrages ou bâtiments des chemins de fer.

Jusqu'aux environs de 1973, les cas sont cependant restés assez limités, mais, pourtant ont souvent donné lieu à des incidents. Ceux-ci étaient dus principalement à l'utilisation d'explosifs par des artisans locaux (carriers le plus souvent) n'ayant aucune notion du tir en site construit. Ces incidents ont amené le plus souvent la SNCF à interdire l'usage de l'explosif sur ses chantiers et à dissuader dans la mesure du possible les riverains de l'utiliser. Elle ne prévoyait dans ses règlements que la protection contre les risques de projections sans envisager les risques d'ébranlements.
Ayant eu connaissance des recherches effectuées dans plusieurs pays (Suède, U.SA etc) et des premières expériences de tir en site construit effectuées en France (RATP, CETE de LYON, etc), la SNCF a constaté que pour une protection efficace de ses constructions, il était préférable de renoncer à une interdiction systématique, et par cela même dangereuse de l'utilisation de I'explosif qui est inévitable pour certains travaux. Cette interdiction conduisait souvent à une utilisation clandestine sans aucun contrôle dans les cas où le tir était indispensable.

La SNCF a donc mis au point un certain nombre de règles définissant les mesures de sécurité à prévoir et en particulier les mesures d'ébranlement. Elle a également, après enquête, agréé un certain nombre d'entreprises spécialisées en minage et un certain nombre de laboratoires de contrôle.

La mise en place de ces règles a coInncidé avec un développement considérable de l'utilisation d'explosifs, tant sur ses propres chantiers que sur des chantiers de riverains. Ce développement s'est fait concurremment avec celui de l'emploi d'engins de déroctage puissants tels que les brise-roches hydrauliques dont les effets sismiques sont souvent plus importants même que celui de l'explosif

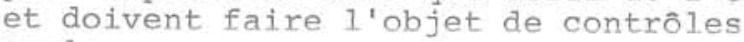
analogues. 
Les raisons de ce développement sont, pour certaines, bien connues : augmentation du cout de la main-d'oeuvre, disparition de la main-d'oeuvre qualifiée pour les travaux pénibles, etc. Il faut ajouter à celles-ci, en ce qui concerne l'exnlosif, l'institution du CPT (certificat pratique de tir). Celui-ci présente l'avantage de donner aux mineurs de travaux publics la connaissance des règles élémentaires de sécurité et de manipulation des explosifs. Il a cependant été largement distribué, après formation par des organismes pour lesquels l'utilisation de l'explosif en site construit n'est qu'une préoccupation accessoire. Ceci a abouti à mettre en place dans les entreprises un grand nombre de préposés et à développer l'usage de l'explosif. Certains de ces préposés, se croyant spécialistes, peuvent amener en site construit les entreprises à prendre des risques importants.

\subsection{Les différents risgues encourus}

Nous n'insisterons pas sur le risque de projections qui est spécifique du tir à I'explosif et aux procédés similaires (Cardox, Airdox, etc). La prévention de ce risque est un problème de protections que la SNCF prévoit dans ses marchés ou dans les conventions avec les tiers.

Le risque essentiel est celui des dégâts causés par les ébranlements, dégâts qui se traduisent par la fissuration des structures des ouvrages, 1'ouverture des fissures ou des microfissures préexistantes et peuvent aller jusqu'à I'éboulement de parties d'ouvrages.

Ce risque existe en cas d'utilisation d'explosifs mais également en cas d'utilisation d'engins mécaniques puissants tels que les vibrofonceurs, les engins de battage les engins de foration, les engins d'abattage, etc. Il faut souligner qu'il est très important en cas d'utilisation de brises roches hydrauliques lourds.

Ce risque est en général évalué à partir de la vitesse particulaire de vibration mesurée sur les structures, critère le plus souvent admis. La valeur de cette vitesse $n^{\prime}$ est cependant pas suffisante pour évaluer les risques et lors de la fixation des seuils admissibles il faut aussi tenir compte d'autres facteurs :

- répétitivité des ébranlements (tirs de carrières, engins mécaniques) et phénomènes de fatigue,

- prédominance de basses fréquences entraînant à vitesse égale des déplacements importants (engins mécaniques),

- phénomènes de résonance.

\subsection{Les installations concernées}

L'importance des installations et du patrimoine immobilier de la SNCF fait que les constructions à protéger sont d'une très grande diversité. On peut citer par exemple: - des immeubles : gares, habitations, etc, - des ouvrages d'art : tunnels, viaducs, ponts, murs de soutènement, parois rocheuses, remblais, etc

- des installations électriques ou électroniques : postes de transformation, postes de signalisation, salles d'ordinateurs, etc - les voies proprement dites, électrifiées ou non, avec les caténaires, les câbles ou fils de signalisation, les ouvrages d'assainissement, etc.

Il importe en outre cue la SNCF s'assure sur ses propres chantiers de la protection des constructions des riverains qui sont aussi très diverses : immeubles, monuments historiques, ouvrages d'art, etc.

\subsection{Les chantiers SNCF}

Ceux-ci sont également d'une très grande diversité. Les chantiers de travaux neufs récents ou en cours ont été exécutés le plus souvent en pleine nature (terrassements du TGV) mais ont parfois nécessité l'emploi d'explosif en site construit (exécution de puits ou de pieux pour fondations de viaducs). Nous reviendrons ultérieurement sur les chantiers de mise au gabarit ou de réparations de tunnels anciens pour lesquels l'explosif ou des moyens mécaniques puissants sont utilisés à assez grande échelle.

De nombreux chantiers de démolition de ponts sont également constamment réalisés, en vue de leur remplacement, tant pour des problèmes de mise au gabarit que de dégradation des ouvrages existants. Nous pouvons citer également le cas de démolitions de constructions anciennes dans les emprises SNCF (châteaux d'eau, silos, blockhaus, etc).

L'explosif ou le brise roches sont aussi utilisés dans le cadre de rectifications d'ouvrages, dans des conditions assez voisines de celles du travail en tunnel ancien. Un exemple particulièrement spectaculaire de ce type de travaux a été la démolition par plots et le remplacement des longrines en béton armé supportant la crémaillère du pont levis de la Bordigue à SETE, réalisée par phases à la faveur de quelques heures d'interruption des circulations.

On peut citer également la destruction assez fréquente de blocs rocheux instables surplombant les installations SNCF. La démolition à l'explosif d'un rocher de $300 \mathrm{~m} 3$ surplombant à la fois la tête d'un tunnel et des immeubles en pleine ville de VIENNE en est un des exemples parmi les plus caractéristiques.

L'explosif est utilisé également dans de nombreux autres cas, création de galeries de drainage, création de passages inférieuns en remplacement de passage à niveau, etc. La figure 1 illustre de façon schématique un des cas de chantiers des plus délicats auxquels la SNCF ait eu affaire, celui de 
la couverture des voies dans la tranchée NEZIN, à côté de la gare de CHAMBERY, en pleine ville.

Les déroctages à l'explosif ont été effectués au-dessus des voies en service, sous des immeubles et en face d'immeubles. Aucun incident ni aucune plainte sérieuse des riverains n'ont été pratiquement notés.

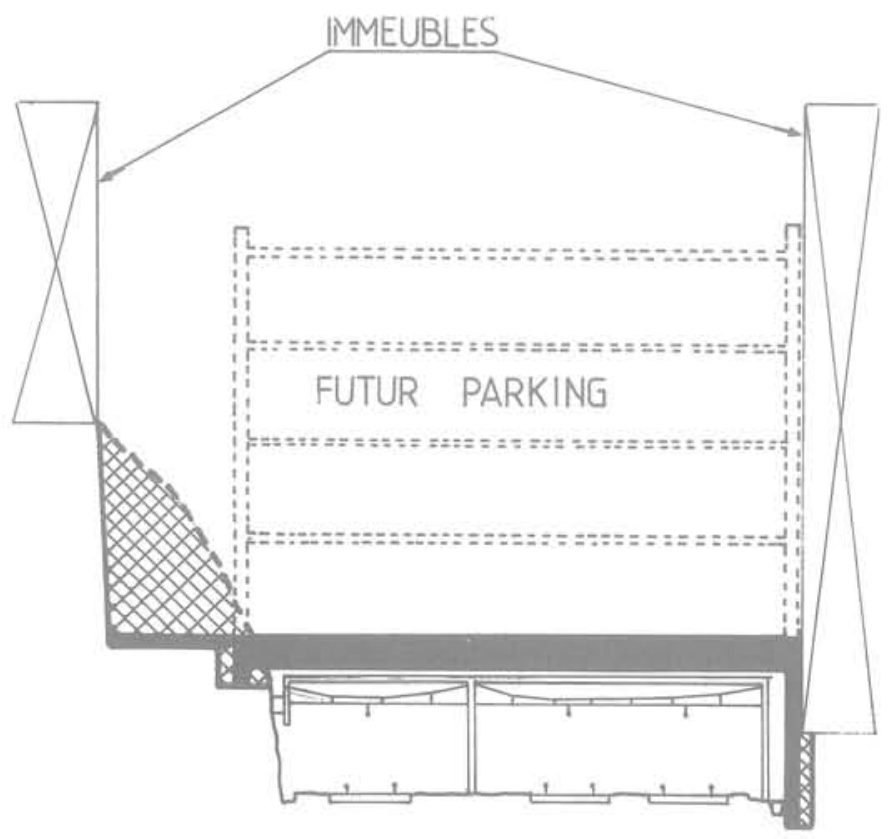

ZONE A DEROCTER

Fig. 1 - Chantier de couverture des voies Tranchée NEZIN à CHAMBERY

\subsection{Les chantiers de tiers à proximité d'ouvrages SNCF}

Les exemples sont trop nombreux pour pouvoir tous les citer, des chantiers de tir se déroulant pratiquement en permanence dans diverses régions. Nous pouvons en donner quelques exemples parmi les plus caractéristiques et récents.

- Travaux de déviation ou rectification de routes par les Directions Départementales de l'Equipement à proximité de tunnels, de viaducs, de murs de soutènement ou autres constructions SNCF. La figure 2 montre le cas d'une rectification de la RN20, à côté de FOIX, à proximité du tunnel de ST PAUL-ST ANTOINE.

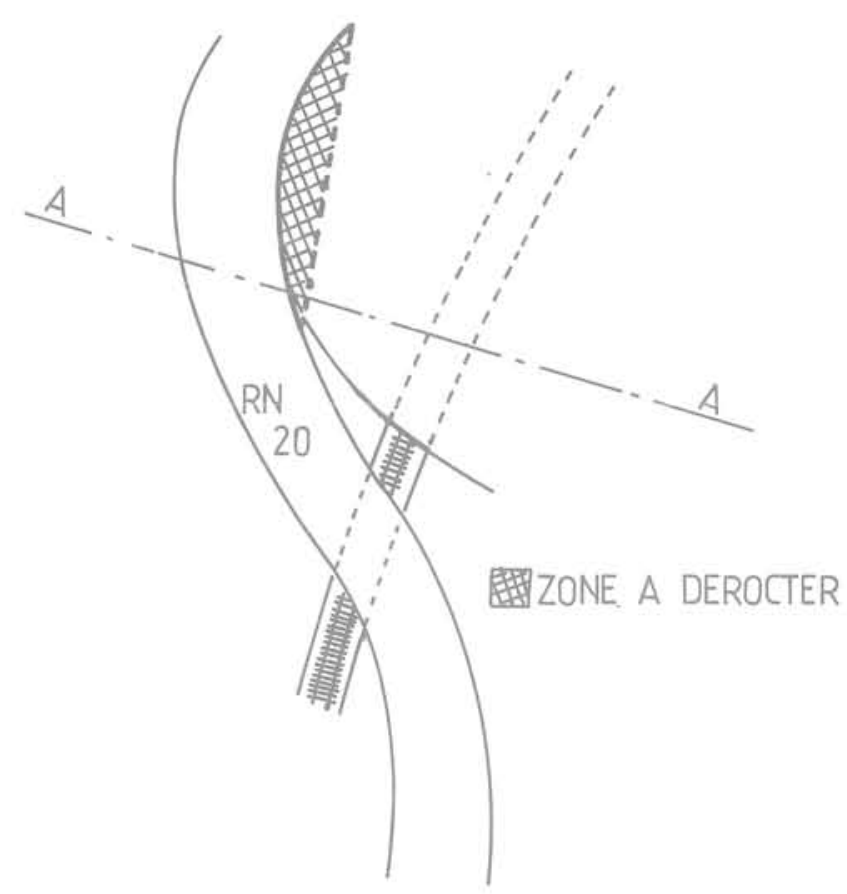

COUPE AA

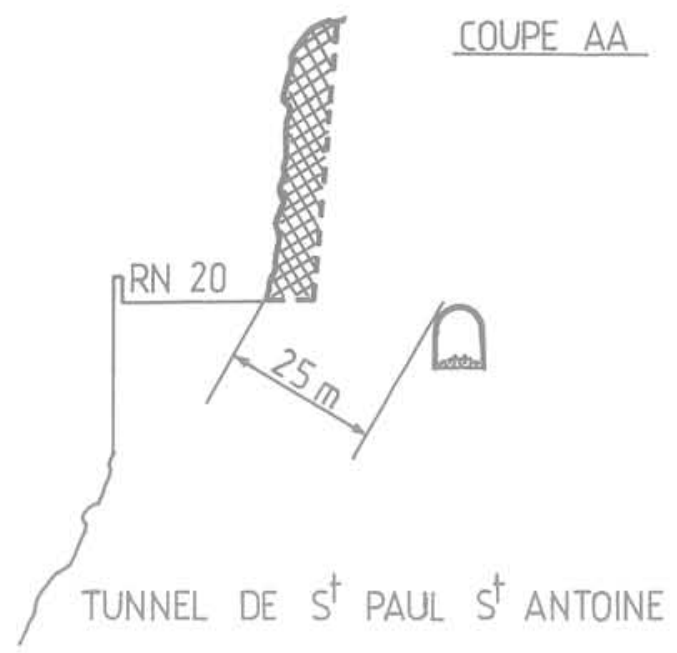

Fig. 2 - Rectification d'un tournant de la RN2O à proximité d'un tunnel

Des chantiers de ce type sont exécutés en permanence à proximité des constructions SNCF.

- Exécution de galeries, collecteurs, fonçages, puits nour différents usages (drainage, acqueducs, oléoducs, conduites diverses, etc). La figure 3 montre l'exemnle d'un chantier de nuits ascenseur et de qalerie à MONACO. Elle illustre un tyoe de chantier très fréquent pour des travaux de tiers et parfois pour des travaux SNCF. Un nombre assez important de chantiers à l'explosif est effectué dans la principauté, en particulier à proximité des ouvrages SNCF. 


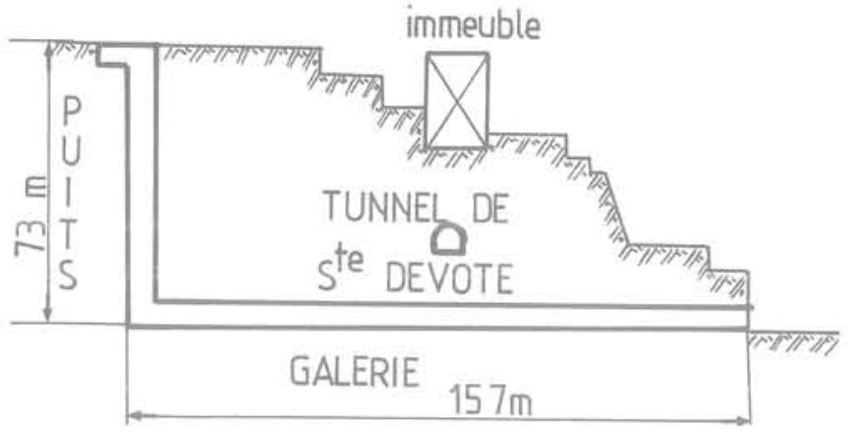

TUNNEL DE $S^{\dagger}$ DEVOTE(MONACO) CREUSEMENT D'UNE GALERIE ET D'UN PUITS

Fig. 3 - Galerie et puits ascenseur de STE DEVOTE à MONACO

- Exécution de travaux de terrassement, nour création de routes ou autoroutes,

d'ouvrages d'art, de fondations ét de bâtiments, etc.

Les figures 4,5 ct 5 illustrent les travaux d'une suppression de passage à niveau avec création d'un nouveau tracé de route en tranchée (travaux DDE) et la création d'un pont-rail (SNCE).

Les figures 4 et 5 montrent la première phase de travaux (DDE) de déroctage de la tranchée rcutière et la deuxième phase de terrassement (SNCF) pour création du pont-rail après déviation provisoire des voies, chantier réalisé en bordure immédiaté de la déviation provisoire et à proximité, outre des installations SNCF, de plusieurs immeubles (hôtel, garage, etc).

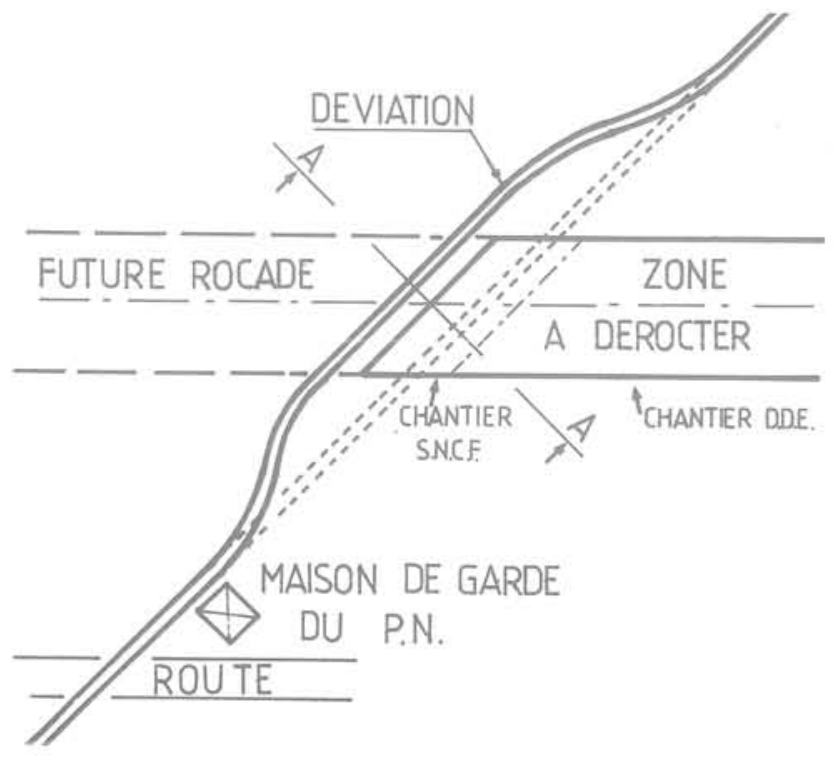

Fig. 4 - Suppression du PNó à AIX-LES-BATNS Premières phases de travaux

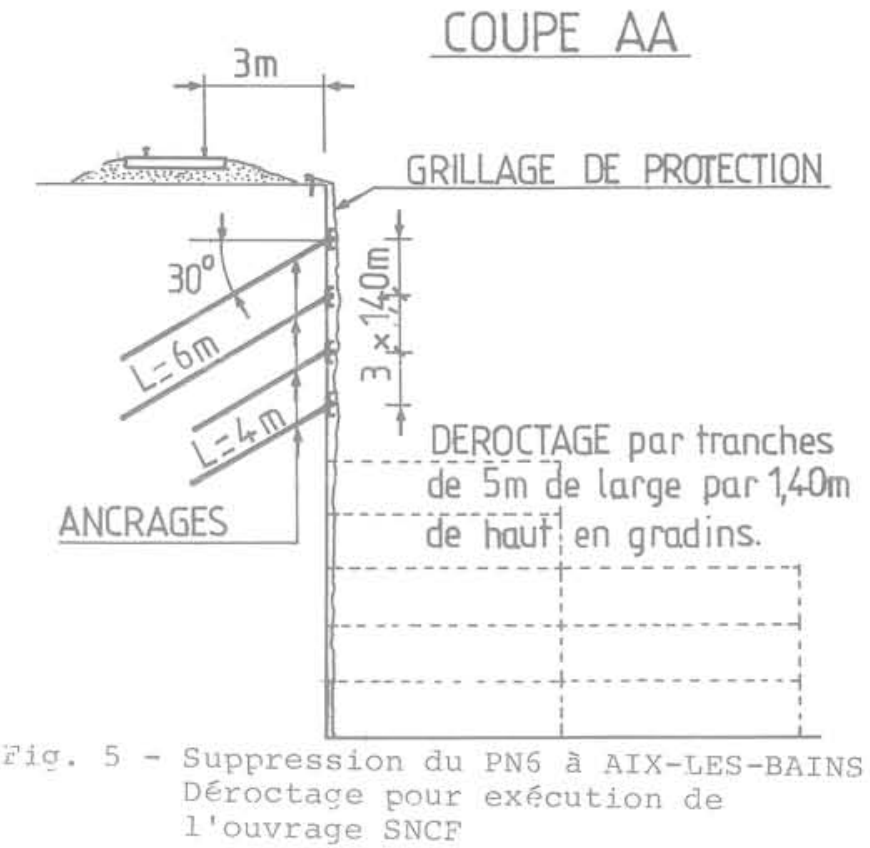

La figure 6 montre la dernière phase de travaux (DDE) avec tir à proximité immédiate de l'ouvrage neuf construit nar la SNCF.
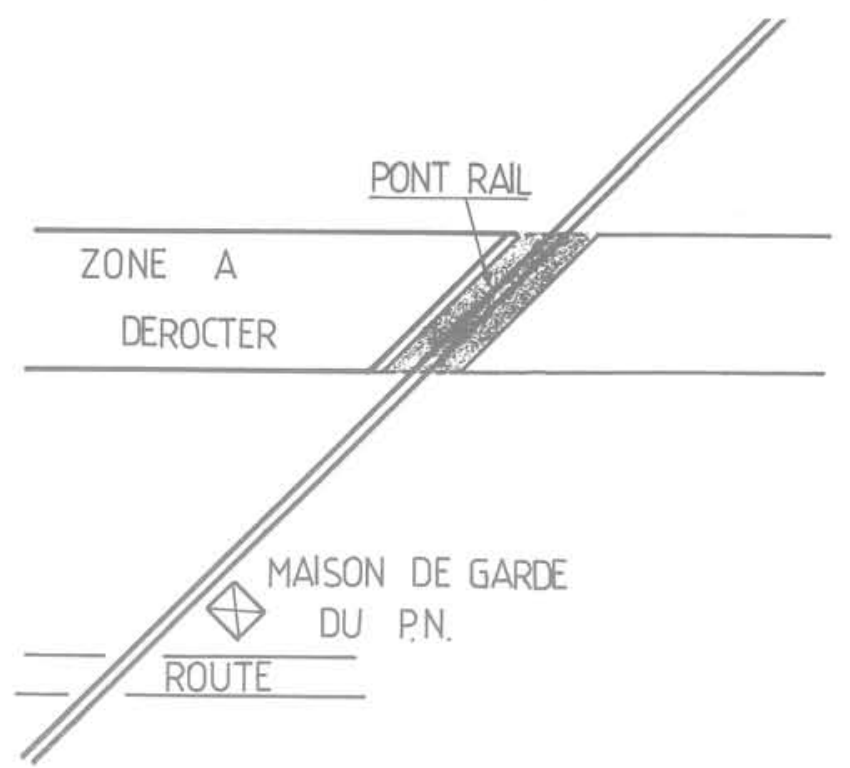

Fig. 6 - Suppression du PN6 à AIX-LES-BAINS Dernière phase de travaux

2 - LES MESURES DE PROTECTION

2.1 Définition des procédures d'essais et de contrôle

Pour ses propres chantiers, la SNCF a été amenée à définir dans ses textes réglementaires applicables aux marchés en complétant les modalités d'applications dans les textes des marchés proprement dits, toute une procédure définissant les précautions à prendre en cas d'utilisation d'explosifs. Les tirs ne peuvent être exécutés que par une entreprise agréée à cet effet par la SNCF. 
Les précautions concernant les mesures de sécurité générale en cas d'emploi d'explosifs et les protections contre les projections doivent être définies par l'entreprise. En ce qui concerne la protection contre les ébranlements, la SNCF demande à l'entreprise de lui soumettre un projet de tir pour essais comprenant un plan de tir d'essai détaillé à essayer dans son ensemble ou par volées partielles, avec contrôle par enregistrement complet des vibrations par un laboratoire agréé par la SNCF. Les seuils de vitesses de vibration et les points de mesure sont définis par la SNCF. Le tir n'est autorisé qu'après visa du plan de tir définitif, mis au point, après ajustement éventuel à l'issue de ces essais.

La SNCF demande en outre maintenant, en cas de présence de constructions appartenant à des tiers que l'entreprise fasse définir les précautions complémentaires à prendre par un laboratoire agréé et qu'elle en tienne compte dans ses projets et plans de tir. Des contrôles sont de plus exigés en cours de chantier, semi-permanents ou permanents par appareils détecteurs de crêtes que l'entreprise doit mettre à la disposition de la SNCF, ponctuels par enregistrements complets effectués par un laboratoire agréé en cours de chantier à l'initiative de la SNCF,

En ce qui concerne les tirs de tiers à proximité des installations SNCF, les précautions à prendre sont définies dans des conventions passées avec les mâtres d'ouvrage concernés. La procédure est très voisine de celle appliquée sur les chantiers $\mathrm{SNCF}$, mais modulée selon le degré de risque encouru par la SNCF. Elles peuvent aller d'une simple demande d'essais et de contrôle effectués par un laboratoire agréé, à une exigence d'exécution des tirs par une entreprise agréée, avec visa des projets de tirs d'essai et plans de tirs par la SNCF. Les exigences de contrôle en cours de chantiers peuvent elles-mêmes être plus ou moins rigoureuses, du simple contrôle par mesure ponctuelle à la mise en place d'appareils détecteurs de crêtes permettant d'actionner la signalisation et d'arrêter les trains en cas de dépassement des seuils fixés par la SNCF.

Les tirs ne sont autorisés qu'en intervalles sans passage de trains (sauf exceptions pour des niveaux d'ébranlements particulièrement faibles). Les dispositifs de nrotection contre les projections sont également définis

\subsection{Détermination de niveaux seuils de vitesse particulaire de vibration à respecter}

Il est impossible de fixer des seuils de vitesse valables dans tous les cas. Outre la modulation en fonction du degré de risques que nous avons évoqué précédemment, $i 1$ faut tenir compte des conséquences possibles de ces risques (importance des circulations SNCF) et des possibilités de surveillance des chantiers (risques de fausses manoeuvres). Il faut également préciser l'emplacement des points de mesure (une vitesse assez élevée peut être mesurée sur un tablier de pont à structure métallique sans conséquences graves alors que la même vitesse mesurée sur les culées peut conduire à des désordres). La SNCF est en conséquence amenée à préciser des seuils fixés après étude de chaque cas particulier.

A titre tout à fait indicatif, les seuils imposés sur des constructions en état moyen ou bon sont pour des tirs effectués en intervalles de circulation de l'ordre de $20 \mathrm{~mm} / \mathrm{sec}$. Dans des cas particuliers, tels que les tirs de carrières, les tirs effectués dans des conditions de circulation importantes de trains etc, ces seuils peuvent être ramenés à une vitesse de $5 \mathrm{~mm} / \mathrm{sec}$. Pour des tirs concernant des installations moins vulnérables, dans le cas de tir en tunnel avec mesure à très courte distance, les seuils peuvent être parfois portés à $50 \mathrm{~mm} / \mathrm{sec}$. Dans le cas d'emplois d'engins mécaniques, tels que les brises roches par exemple, les signes d'apparition de dégâts dus à la fois aux basses fréquences, à la résonance et à la répétitivité des ébranlements amènent à fixer des seuils beaucoup plus bas ( 2 à $5 \mathrm{~mm} / \mathrm{sec}$ selon les cas).

\subsection{Agrément de laboratoires chargés des contrôles}

La SNCF a été amenée à créer, pour les mesures de vibration, un agrément des laboratoires chargés des contrôles. Une dizaine de laboratoires l'ont été jusqu'à présent. Ce sont :

- des organismes publics ou semi publics : - laboratoires du Ministère de l'Environnement, dont certains tels que les CETE de LYON, AIX-EN-PROVENCE et NANCY ont déjà acquis une grande expérience en ce domaine,

- laboratoires du CEBTP (PARIS et MARSEILLE)

laboratoire du BRCM,

- des laboratoires privés pour lesquels la SNCF s'est assurée qu'ils disposent à la fois du personnel compétent et du matériel nécessaire.

\subsection{Agrément des entrenrises de minage spécialisées}

En outre des nombreuses qualifications pour lesquelles la SNCF accorde un agrément aux entreprises spécialisées, trois qualifications nouvelles ont été créées pour

l'emploi de I'explosif :

- le tir en site courant,

- le tir en site construit,

- le tir en tunnel.

Un petit nombre d'entreprises a reçu l'une ou la totalité de ces qualifications. Ce sont :

- les quelques entreprises de minage très en sitécialisées particulièrement pour le tir 
- les services de minage de quelques grandes entreprises ayant pratiqué des travaux particulièrement délicats en site construit.

\section{3 - CAS PARTICULIER DES CHANTIERS DE TUNNELS}

\subsection{Types de tirs effectués - plans de tir}

Le tir en tunnel ancien est utilisé essentiellement pour les travaux de mise au gabarit, le plus souvent pour l'électrifications des lignes (électrification de la rive droite du Rhône : 18 tunnels traités de 1974 à 1978, électrification de NARBONNE PORT-BOU 7 tunnels traités ou en cours de traitement depuis 1978 , etc). Il peut être également utilisé dans des conditions analogues pour des travaux de confortement en surépaisseur des voûtes nécessitant un abaissement du niveau des voies.

La figure 7 montre le type de travaux pour lesquels, outre l'abaissement proprement dit de la plateforme rocheuse, 1'explosif est utilisé :

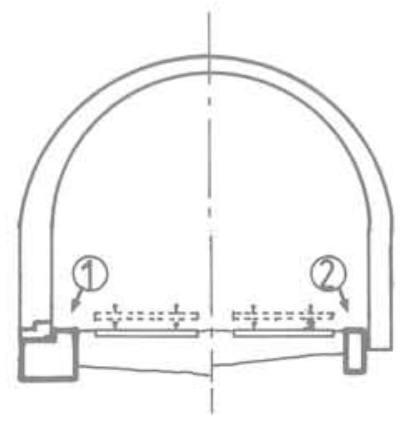

(1)REPRISE EN SOUSCEUVRE DE PIEDROI.

\section{(2) CREATION DE} LONGRINES.

Fig. 7 - Mise au gabarit de tunnels

L'explosif est aussi utilisé, comme l'indique la figure 8 pour 1 'approfondissement ou la création de niches servant de refuges à personnel, couramment effectués lors de travaux d'entretien.

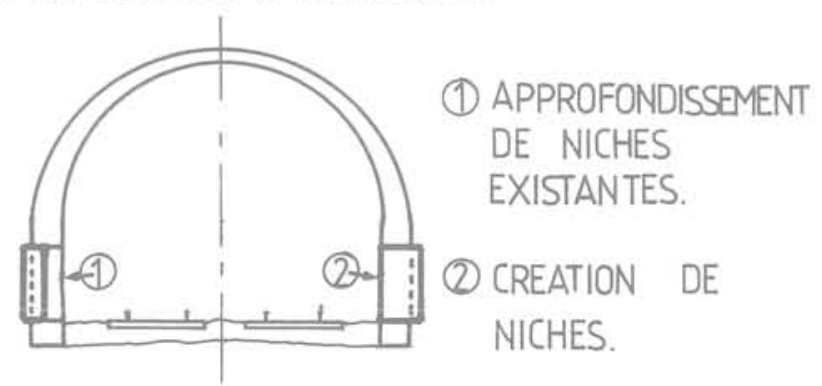

Fig. 8 - Création ou approfondissement de niches refuges pour le personnel

Il peut être également utilisé lors de travaux d'entretien dans des cas divers (démolitions partielles de piédroits, saignées, drainage, etc).

Des plans de tirs appropriés sont utilisés dans chaque cas. Ils sont établis dans le but de réduire le plus possible le niveau de vibrations et sont caractérisés par une maille de foration très serrée et l'utilisation de toute la gamme possible d'amorces à retard ordinaire et microretard de façon à limiter la charge unitaire instantanée qui ne dépasse le plus souvent jamais 200 grammes d'explosif.

La figure 9 donne l'exemple d'un plan de tir de niche.
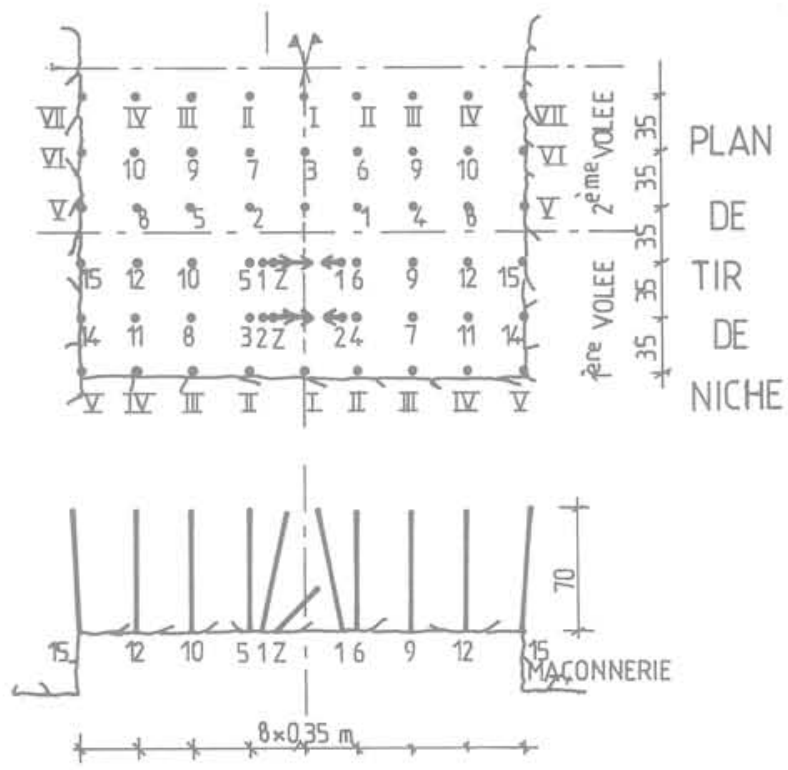

Fig. 9 - Plan de tir de niche

\subsection{La procédure d'essais et de contrôle}

La procédure telle que nous l'avons indiqué précédemment est détaillée par une consigne et précisée dans un texte type inclus dans les marchés. L'emplacement des points de mesure est précisé. La présentation des résultats de mesure est définie. La particularité de ce type de tir est que les maçonneries à protéger se trouvent à proximité immédiate de la source d'ébranlements. Les capteurs sont en général placés à 2,5 et $5 \mathrm{~m}$ de la bordure de la zone de tir. Les plans de tir font l'objet d'une série d'essais avec mesures complètes par un laboratoire agréé sous contrôle de la SNCF. Les plans de tirs définitifs sont visés après adaptations nécessaires. Un contrôle semi permanent ou permanent est ensuite effectué à l'aide d'un détecteur de crêtes étalonné avec la chaine de mesure lors des essais. En cas de dépassement des seuils fixés ou en cas de modifications des conditions nécessitant une adaptation du plan de tir, il est procédé à de nouvelles séries d'essais.

\subsection{Caractéristiques des vibrations enregistrées - Seuils admis}

La nécessité de protéger des maçonneries à proximité immédiate de la zone de tir a amené à exécuter des mesures au voisinage de la source d'ébranlements. Les vibrations enregistrées s'étalent en conséquence sur 
une très large bande de fréquences de 10 à 1000 Hertz environ, les vitesses les plus élevées étant en général produites au-delà de 100 Hertz. Les quelques rares cas de dégâts mineurs relevés lors d'essais se sont produits pour des vitesses dépassant $80 \mathrm{~mm} / \mathrm{sec}$ à des frécruences situées entre 100 et $250 \mathrm{~Hz}$. La vitesse de $50 \mathrm{~mm} / \mathrm{sec}$ que nous adoptons en général comme seuil n'est dépassée que pcur quelques numéros de retard isolés, en général à des fréquences très élevées. Nous avons êtté amenés en conséquence à des analyses détaillées des vibrations produites, ce qui nous conduit à tolérer certains dépassements du seuil de $50 \mathrm{~mm} / \mathrm{sec}$ adopté à condition d'être assurés que ce dépassement se produit à des fréquences dépassant $250 \mathrm{~Hz}$.

M. ALLARD du CETE d'AIX-en-PROVENCE a procédé de son côté à une analyse, en liaison avec nos services. Analyse quj fait $1^{\prime o b j e t ~ d e ~ l a ~ c o m m u n i c a t i o n ~} n^{\circ} 17$. 